Recepción: 15 / 04 / 2017

Ciencias Educativas

Aceptación: 01 / 05 / 2017

Revisión de Literatura

Publicación: 15 / 05 / 2017

\title{
EI desarrollo y sostenibilidad de las competencias profesionales en los estudiantes
}

\author{
Development and sustainability of professional skills \\ on students
}

\section{Desenvolvimento e sustentabilidade de habilidades profissionais em estudantes}

Evelyn J. Henríquez-Antepara ' evelyn.henriqueza@ug.edu.ec

Ruth E. Paredes-Santín " ruth.paredess@ug.edu.ec

Oscar O. Apolinario-Arzube ${ }^{\mathrm{II}}$ oscar.apolinarioa@ug.edu.ec

Correspondencia: evelyn.henriqueza@ug.edu.ec

\footnotetext{
Magister en Docencia y Gerencia en Educación Superior; Profesora de Segunda Enseñanza Especialización Comercio Exterior; Licenciada en Ciencias de la Educación Especialización Comercio Exterior; Universidad de Guayaquil, Ecuador. Licenciado en Ciencias de la Educación mención Ingles, Universidad de Guayaquil, Ecuador. Magister en Administración de Empresas mención Recursos Humanos y Marketing; Licenciado en Sistemas de Información; Analista de Sistemas; Universidad de Guayaquil, Ecuador.
} 


\section{Resumen}

En los últimos años en sentido general uno de los objetivos fundamentales de los modelos educativos que respalda la Educación Superior, se direcciona al desarrollo de las competencias profesionales en los egresados de las diferentes carreras; precisamente por la importancia que estas tienen en el desempeño de los graduados, una vez que son insertados en el mercado laboral donde deben mostrar sus competencias con la mayor optimización posible. En correspondencia con esto se pretende tener una aproximación a aspectos esenciales que hoy caracterizan la realidad ecuatoriana y mundial en cuanto al desarrollo y sostenibilidad de las competencias profesionales de los egresados de las diferentes carreras. Particular interés tiene el caso de la carrera de Ingeniería en Sistemas Computacionales" de la Universidad de Guayaquil, donde se evidencia la existencia de una crisis en las competencias profesionales de los alumnos que egresan. El artículo que se presenta está basado en la búsqueda y la reflexión científica unido a vivencias prácticas que han posibilitado corroborar la presencia de una problemática que dada su importancia necesita de una mirada más profunda que permita en breve tiempo transformar esta situación y convertirla en oportunidad.

Palabras clave: Educación Superior; competencias profesionales; carrera de ingeniería en sistema; crisis; oportunidad. 


\begin{abstract}
In the last few years, in general, one of the fundamental objectives of the educational models that support Higher Education, is directed to the development of the professional competences in the graduates of the different careers; precisely because of the importance they have in the performance of graduates, once they are introduced in the labor market where they show their skills with the highest possible optimization. Therefore, it is expected to establish an approach to the essential aspects that today characterize the Ecuadorian and worldwide reality in terms of the development and sustainability of the professional skills of graduates of different careers. Particularly, the case of the Engineering Degree in Computational Systems of the University of Guayaquil, where there is evidence of a crisis in the professional competences of students who graduate. This article is based on research and scientific reflection coupled with practical experiences that have made it possible to confirm the presence of a problematic that due to its importance needs for a deeper look that allows in a short time to transform this situation into an opportunity.
\end{abstract}

Key words: Higher Education; professional skills; system engineering career; crisis; opportunity. 


\section{Resumo}

Nos últimos anos, em geral, um dos objectivos fundamentais dos modelos educativos que apoiam o Ensino Superior é dirigir-se ao desenvolvimento das competências profissionais dos licenciados das diferentes carreiras; Precisamente por causa da importância que têm no desempenho dos graduados, uma vez que são introduzidos no mercado de trabalho onde eles mostram suas habilidades com a maior otimização possível. Por tanto, espera-se estabelecer uma abordagem aos aspectos essenciais que hoje caracterizam a realidade equatoriana e mundial em termos de desenvolvimento e sustentabilidade das competências profissionais de graduados de diferentes carreiras. Particularmente, o caso da Licenciatura em Engenharia em Sistemas Computacionais da Universidade de Guayaquil, onde há evidências de uma crise nas competências profissionais dos alunos que se formam. Este artigo é baseado em pesquisa e reflexão científica, juntamente com experiências práticas que tornaram possível confirmar a presença de uma problemática que, devido à sua importância, precisa de um olhar mais profundo que permita em pouco tempo transformar esta situação em oportunidade.

Palavras chave: Ensino superior; habilidades profissionais; engenharia de sistemas; crise; oportunidade. 


\section{Introducción.}

Los cambios que trajo la globalización sin duda alguna intervienen en la educación y su influencia en la economía de cada nación, la tecnología e innovación en los diversos ámbitos empresariales exigen una demanda de profesionales capacitados en todas las competencias tanto genéricas como específicas.

Actualmente los modelos educativos apuntan cada vez más al desarrollo y sostenibilidad de las competencias profesionales en los egresados, precisamente porque estas garantizan entre otros aspectos mayor autonomía, eficiencia, optimización y calidad en cada proceso y por consiguiente en cada egresado.

A nivel mundial el desarrollo de las competencias profesionales se ha caracterizado por ocupar un lugar fundamental dentro del contexto de la educación superior, por ejemplo en países como: España, Holanda, Francia, México y países de América Latina se puede apreciar cómo estas ocupa un lugar fundamental ya que se debe formar recursos humanos preparados para enfrentar las nuevas necesidades.

En Ecuador, este proceso ha transitado por diferentes etapas hasta llegar a la actualidad, donde los modelos educativos existentes buscan no solo desarrollar las competencias profesionales en los alumnos, sino también encontrar alternativas que las conviertan en sostenibles y en verdaderas oportunidades; entendidas como mayor calidad en el desempeño profesional. Sin embargo, alcanzar este estadio requiere de una mirada más profunda al fenómeno en cuestión, supone en primer orden la ruptura de las crisis que se originan a partir de las características de la propia situación y de lo que es más importante aún el análisis y rediseño de los diferentes currículos. 
¿Cómo lograr que el desarrollo y sostenibilidad de las competencias profesionales de los egresados de las diferentes carreras se transforme de una crisis en una oportunidad?, es hoy una interrogante y una problemática a resolver, máxime si tenemos presente que el mundo moderno y globalizado en el cual nos desenvolvemos exige de un profesional cada vez más competente y eficaz.

Dada la importancia del tema, el trabajo que se presenta intenta tener una aproximación teórica y reflexiva que en un primer momento nos permite develar qué ocurre en el contexto del desarrollo y sostenibilidad de las competencias profesionales en sentido general en las diferentes carreras, con particular interés en la Carrera de Ingeniería en Sistemas Computacionales, Facultad de Ciencias Matemáticas y Físicas de la Universidad de Guayaquil,

En la misma medida que en una segunda parte se aportaran posibles soluciones a tener en cuenta para que la actual crisis se convierta en oportunidad.

Antes de comenzar a revisar dichos avances hacia nuestra investigación se debe reflexionar y meditar sobre el tema a plantear, principalmente enfocarse en la actualidad de nuestro entorno educativo, especialmente a las demandas de ofertas laborales. Se profundizará y se dará un enfoque sobre las razones del descenso educativo, probablemente la falta de aptitud profesional para resolver un problema y desenvolverse entre la influencia que existe en el mercado o el dinamismo entre las ofertas de las empresas; otra razón seria la poca motivación por parte de los docentes a los alumnos en tener una mentalidad positiva y competente que le permita tener un excelente desenvolvimiento en sus funciones como profesional.

Con esta problemática, se debe corroborar la magnitud de las influencias que afectan a los futuros profesionales, tal vez sea la falencia en el declinamiento hacia otras ofertas que demanda la 
sociedad; nuevos mercados que sobresalen por ende al no destacarse, influyen en el esfuerzo y motivación en los planteamientos del conocimiento para así tener el enfoque requerido.

Con el cambio radical en los Programas Educativos una alta gama de conocimiento se manifiesta, debido al avance de la tecnología que va de la mano del esfuerzo investigativo. Pero si esto no es guiado de forma asertiva, los resultados serían negativos y no tendríamos profesionales que se ajusten ante la exigencia de la sociedad actual. Se necesita que la fuentes de aprendizaje sean actualizadas, dentro y fuera de la Universidad de Guayaquil para ver el desarrollo de los futuros prospectos o candidatos a la rama de profesión que se está abarcando; debemos de recaudar información, y con las misma comparar si las bases de conocimiento de la facultad que se implementa en los futuros profesionales de esta prestigiosa entidad educativa concretará con el bienestar y el desarrollo autónomo que presenta las demás instituciones educativa del país, como gestores de profesión con el nivel de conocimiento que les permita enfrentarse a diario a las diversas situaciones en el mundo laboral.

El perfil profesional de la carrera de Ingeniería en Sistemas Computacionales es ofertar a la sociedad ingenieros que sean capaces de ocupar puesto administrativos y gerenciales; antagónicamente los egresados no se encuentran preparados para desempeñar cargos en los mando medios, donde tienen como tarea diaria tomar decisiones, ser creativos, manejar grupos y adaptarse a los diferentes ambientes laborales.

Más allá se pretende que el Ingeniero en Sistemas Computacionales, sea capaz de crear fuentes de empleos para dinamizar la economía del país, pero la realidad es otra, ellos presentan algunas falencias en la competencias genéricas, ven con gran preocupación y temor que se acerque la hora de realizar su tesis, pues carecen de herramientas de redacción, fundamentos básicos para 
hacer una investigación científica; no poseen habilidades para hablar en público, lo que les dificulta al momento de sustentar de su tesis, donde deben defender el tema y el trabajo realizado requisito final para incorporarse como ingenieros, tomando como referencia principios claros como: "lo sé pero, no lo sé decir" tienen el conocimiento que han adquirido durante los ocho semestres de estudios, pero no tienen desarrollada la competencia lingüística oral y escrita, que les permita transmitir el mensaje correcto y tener un desenvolvimiento óptimo.

Es común que al realizar un trabajo en grupo en el que solo participen de forma activa la tercera parte, ya que están identificados los roles entre los estudiantes, saben quién es bueno para investigar, para exponer y quien se encarga de liderar el grupo, esto perjudica al resto de integrantes que no les permite exigirse y desarrollar sus habilidades, creando malestar entre los docentes cuya meta es alcanzar en los alumnos un aprendizaje significativo, pero no tienen los fundamentos para hacerlo, en las asignaturas que les permiten el desarrollo de las competencias genéricas como: Idioma Español, Comunicación Oral, Metodología de la Investigación que se imparten en el primer y segundo semestre no se incentivó el desarrollo de dichas habilidades, la metodología tradicional que utilizan los docentes que se limita de forma teórica, la cual solo se quedó en el cuaderno de apuntes o en algún dispositivo de almacenamiento.

Otra problemática es la carencia de valores en los estudiantes, la ética, el respeto por los derechos de autor; es un elemento que ellos no consideran al momento de realizar sus trabajos de investigación, es común que al revisar los ensayos que la mayoría no tienen idea de lo que está escrito en su deber, es un trabajo de "copy and paste" entonces si el estudiante no lee las fuentes de donde extrajo la información y no realiza un análisis emitiendo su conclusión ¿Cuál es el objetivo del trabajo? Los beneficiados son los locales de impresión que están situados dentro y fuera de la carrera, se ha creado una idea errónea de la investigación a través del internet, se debe fomentar a 
una búsqueda basada en la selección de netgrafía académica, para que el estudiante después de leer emita su criterio y así compartirlo en la clase creando círculos de aprendizaje.

Falta motivación en el estudiante, es fácil entender y comprender que ellos desean obtener su título, para apostar por una mejor plaza laboral, pero simplemente es la llave que abre la puerta de una institución por grande o pequeña que sea, su permanencia la garantiza el desarrollo óptimo de las actividades asignadas. Se evidencia desinterés por parte del alumnado, existen docentes que aún se aferran a la metodología tradicionalista creando malestar en el estudiante que se limita a ser un simple escucha, deja al estudiante en una zona de confort que luego le pasará factura.

Los estudiantes manifiestan que la carrera carece de una adecuada infraestructura y de suficientes laboratorios para poder realizar sus prácticas, están conscientes que la institución hace lo mejor posible con los recursos que posee, pero ellos deben contribuir con un autoaprendizaje y especializarse en un área, lo cual le permitirá potencializar los conocimientos que se adquieran en las clases, algunas asignaturas no siguen una secuencia creando descoordinación en el proceso de aprendizaje y desarrollo de las competencias específicas .

Otro debilidad que se encuentra en los estudiantes es la falta de análisis y reflexión lo que le impide expresar sus opiniones con argumento valederos, falta construcción en los procesos del pensamiento, los estudiantes en su mayoría son jóvenes inseguros, con desventaja frente a unos pocos que si desarrollaron un pensamiento crítico y reflexivo.

Estamos de acuerdo con lo manifestado por la Dra. Elizabeth Larrea (Larrea, 2016) en el artículo "Modelo de la Formación Integral Orientado a la Investigación y Gestión Social del Conocimiento" aprobado por es OCAS (Órgano colegiado Académico Superior) el 25 de febrero del 2016, lo que le permitió adoptar el modelo educativo Ecológico. 
La UG como Universidad ecuatoriana debe enfrentar y afrontar los retos de un nuevo marco regulador de la Ley Orgánica de Educación Superior y nuevos procesos en el ámbito organizativo, académico y curricular, a la par que América Latina y el mundo, y asumir una realidad compleja y cambiante dentro de la vorágine de avances en ciencia y tecnología, los contextos de inequidad e incertidumbre, las dificultades en nuestras dinámicas histórico-culturales. Los cambios que enfrenta la UG, nos hacen reflexionar y asumir la oportunidad de proponer un proceso organizativo en la formación y multiplicación espacios de aprendizaje e investigación, en prospectiva a lograr la expansión del conocimiento y con la visión de que aprovechando el cambio paradigmático en las formas de producción del conocimiento, la universidad logre un posicionamiento global interactuando con los avances científico tecnológicos mundiales con el horizonte puesto en el beneficio del país. (Larrea \& Montalvan, 2016)

Analizando lo expuesto surgen las siguientes interrogantes en torno a las competencias profesionales de los estudiantes de la Carrera de Ingeniería en Sistemas Computacionales:

¿Qué está sucediendo con los estudiantes de la carrera de Ingeniería en Sistemas Computacionales en el desarrollo de las competencias profesionales genéricas y específicas? ¿Será que se debe revisar la malla y actualizarla de acuerdo a las nuevas exigencias de la sociedad laboral? ¿El modelo ecológico que plantea la Universidad de Guayaquil se ve reflejado en el currículo? ¿Los proyectos de aulas deben ser más participativos y cambiando el rol de sus integrantes? El trabajo final para aprobar el semestre debe ser articulado con algunas asignaturas? ¿Se encuentra articulada la Malla Curricular de la Carrera? ¿La metodología que utilice el docente debe estar orientada al desarrollo del pensamiento crítico y creativo? 
Evelyn J. Henríquez-Antepara; Ruth E. Paredes-Santín; Oscar O. Apolinario-Arzube

Estas y otras interrogantes surgen y se manifiestan tanto en estudiantes como docentes, sin encontrar la totalidad de las respuestas. Lo cierto es que estas definen puntos críticos dentro del sistema educativo que se desarrolla actualmente en la Carrera de Ingeniería de Sistemas Computacionales.

Se debe aceptar la dificultad de asumir de forma integrada las diferentes perspectivas de análisis porque la competencia profesional se suele ver como la sumatoria de capacidades individuales singulares específicas, o como un ejercicio de raciocinio técnico definido a priori, de modo prescriptivo (es decir, una manera de aplicar conocimientos para solucionar problemas prácticos). Se quiere ir en contra de la corriente que tiende a infravalorar la experiencia profesional como metodología constructiva, cuando en realidad la experiencia adquirida en el trabajo constituye una buena base para el crecimiento de una mentalidad heurística, investigadora, núcleo central de una mentalidad competente, que va a servir para usar la pesquisa y la resolución de problemas en cualquier situación labor. (Moreno Moreno \& Muñoz García, 2007)

\section{Antecedentes}

En década de los noventa aparecen los proyectos de reforma curricular a gran escala que buscan adaptar de modelos académicos que respondan a determinadas demandas educativas. Enmarcándose la mayoría de los proyectos en políticas educativas que surgen del contexto de la globalización económica, acuerdos comerciales y comercio internacional para conseguir la certificación y homologación de los programas educativos.

La UNESCO, señala que las nuevas generaciones del siglo XXI, deberán estar preparadas con nuevas competencias y nuevos conocimientos e ideales para la construcción del futuro, por lo que la Educación Superior entre otros de sus retos se enfrenta a la formación basada en las 
competencias y la pertinencia de los planes de estudio que estén constantemente adaptados a las necesidades presentes y futuras de la sociedad para lo cual requiere una mejor articulación con los problemas de la sociedad y del mundo del trabajo (1998:1-4).

Tomamos lo expuesto como referente en el planteamiento y desafíos de las Instituciones de Educación Superior y la influencia de sus resultados en la sociedad y economía de cada nación.

Desde esta perspectiva de innovación curricular, surge la educación basada en competencias. Modelo que al igual que el currículo flexible, calidad total, reingeniería educativa, planeación curricular estratégica, análisis institucional prioriza las ideas de eficiencia, calidad y competitividad y se encuentra asociado con modelos de certificación y evaluación de la calidad educativa o profesional y a la búsqueda de la homologación entre planes de estudio de diversas instituciones, entidades y países (Diaz Barriga, 2003)

El reto que tienen las universidades es cada vez más complejo, el de formar profesionales eficientes y competentes para una sociedad y economías globalizadas, exige que la instituciones este vinculadas con el mundo laboral para conocer las nuevas necesidades y realizar los cambios en los modelos curriculares, la implementación de nuevas estrategias metodológicas que les permita ser transmisoras de competencias genéricas y específicas para lograr atender la necesidades de presentes y futuras de las empresas.

En este orden de pensamientos nos resultan extremadamente interesantes las ideas de (Noro, 2016), cuando comenta que... "La prioridad de un país es tener el mejor sistema educativo y escuelas más eficientes, para ofrecer y lograr la mejor educación para todos los ciudadano. Refiere que Universidad y calidad educativa son principios que no se pueden discutir," Apunta también, que... “el desarrollo de un país depende de sus Universidades, allí está el conocimiento, la 
investigación, los profesionales, el pensamiento. Sin buenas Universidades no se puede pensar en un país acorde a la sociedad del conocimiento..." A lo anterior nos atrevemos a añadir que no existe transformación social de un país sin que se tenga en cuenta el papel de las Universidades, como centros gestores no sólo de conocimientos, sino también de innovaciones científicas y sociales.

En 1998 surge en un contexto de reflexión sobre la educación superior el proyecto Tuning Educational Estructures in Europe "afinar estructuras educacional en Europa, parte de una premisa que el área social y económica europea debe de ir en paralelo con el área de educación. Inician este proceso cuatro ministros de educación superior Reino Unido, Francia, Italia y Alemania quienes reunidos en la universidad de Soborna en Paris, Francia analizan que frente a los cambios en los entornos educativo y laboral que conllevan a la diversificación de carreras profesionales, las Universidades tienen la obligación de entregar a los estudiantes y a la sociedad en conjunto, un sistema de educación superior que brinde las mejores oportunidades para buscar y encontrar en ámbito de excelencia laboral. Los ministros participantes exhortan a los demás países miembros de la UE y universidades europeas a que se consolide la presencia de Europa en el mundo a través de la educación continua y actualizada que ofrece a sus ciudadanos.

En el 2010 se proclama de necesidad de crear un “ Espacio Europeo de Educación Superior “ esta organización sigue los principios de calidad, movilidad y competitividad para mejorar la nivel de empleo en la Unión Europea y convertir el sistema de formación como un foco de atracción para estudiantes y docentes para el resto del mundo.

En este proceso surge el proyecto "6X4 UEALC" que hace mención a seis profesiones (Administración, Ingeniería Electrónica, Medicina, Química, Historia y Matemáticas) en cuatro ejes 
(competencias profesionales, créditos académicos, evaluación y acreditación y formación para la innovación y 1 investigación)

En el 2003 se presenta a la Comisión Europea como un trabajo intercontinental debido a que es un proyecto que retoma experiencia europea y es enriquecido por aportaciones académicas tanto europeas como latinoamericanas.

El proyecto Alfa Tunning América Latina aparece en el 2005 en Buenos Aires donde el grupo de trabajo elaboraron una lista de 27 competencias genéricas, actualmente este proyecto está conformado por 62 universidades latinoamericanas de los 18 países; Argentina, Brasil Chile, Colombia, Costa Rica, Cuba, Ecuador, El Salvador Guatemala, Honduras, México, Nicaragua, Panamá, Paraguay, Perú, Uruguay y Venezuela.

En el Ecuador la crisis en la educación superior era evidente, se inician evaluaciones que el gobierno de turno realizó a las Instituciones Educativa, para promover la calidad según (CONEA, 2009):

El Mandato Constituyente No. 14 hace referencia al nivel que estableció el CONEA para concienciar la excelencia y maximizar la enseñanza en cada institución la misma que garantizará el desempeño ético y profesional del universitario. Mediante un estudio riguroso se estableció las medidas y clasificación de cada Institución, la cual está enfocado en cuatro puntos muy importantes que a continuación se detallan: - “(..) academia, estudiantes y entorno de aprendizaje, investigación y gestión interna" - (CONEA, 2009).

Gracias a los puntos que destacan ha tenido gran acogida por parte de las Instituciones que denotan el cumplimiento sobre las infraestructuras de enseñanza y el privilegio de incentivar a 
estudiantes en el desarrollo del entorno practico e investigativo, cuyas acciones, el docente establecerá como metodología del desarrollo cognitivo y autónomo, según lo demuestra el LOES en los diferentes artículos que dispones para una educación de calidad.

\section{Universidades en la categoría $B$}

\begin{tabular}{|l|}
\hline UNIVERSIDAD AGRARIA DEL ECUADOR \\
UNIVERSIDAD DE ESPECIALIDADES ESPIRITU SANTO \\
\hline UNIVERSIDAD DE GUAYAQUIL \\
UNIVERSIDAD DE LAS AMERICAS \\
UNIVERSIDAD ESTATAL DE BOLIVAR \\
\hline UNIVERSIDAD NACIONAL DE CHIMBORAZO \\
\hline UNIVERSIDAD NACIONAL DE LOJA \\
\hline UNIVERSIDAD POLITECNICA SALESIANA \\
\hline \\
UNIVERSIDAD TECNICA DEL NORTE \\
\hline
\end{tabular}

“Cuadro dado por (CONEA, 2009)”

Pero el análisis que realizó el SENESCYT durante el año 2012 sobre varias irregularidades que implicaba a la Universidad de Guayaquil a estudiantes, al denotar ciertas falencias por parte académica y administrativa, es decir denuncia hechas a la Institución sobre cobros inadecuados y visto de manera indebida, cuyo resultado a notar esto dedujo que (CES, 2014):

“La Comisión de Investigación para la Universidad de Guayaquil, remitió para conocimiento y resolución del Pleno del CES el informe sobre la Universidad de Guayaquil, el cual incluyó dentro de sus conclusiones finales las siguientes: 
a) Existencia de irregularidades en el cumplimiento del Principio de Gratuidad.

b) Existencia de irregularidades en la conformación del máximo órgano colegiado de la Universidad.

c) Existencia de Irregularidades de carácter académico, administrativo y financiero.” - (CES, 2014)

Pero a pesar de esto, la Universidad de Guayaquil ha extremado medidas ante la situación que estaba aconteciendo, en especial, sobre el caso CES, que hace referencia a las diferentes irregularidades que se suscitaba en aquellos tiempos, en fin, uno de los casos que llama la atención: "Que sucedía con los estudiantes que estaban postulados a ser profesionales en aquellos tiempos, sobretodo cuan cuantificable y cualificable estaban saliendo al mercado laboral con dichos conocimientos ante la crítica que se denotó por parte de la sociedad”.

Ante la increíble negligencia que denota la Institución, con las severas acusaciones por parte de los estudiantes, con ello se verificó que ya venía sucediendo durante varios periodos, increíblemente no había medidas preventivas o correctivas para los futuros profesionales que incursionaban sus estudios en la Universidad de Guayaquil, como concienciar la modificación de los métodos o enseñanza educativa, aunque se sabe, que esta metodología iba a la par de la evolución de la tecnología, pero para ello se concretó un acuerdo sobre la re-infraestructura de la Universidad durante un breve lapso que gestionó las Autoridades del Gobierno de Turno para ver si los cambio efectuados serian de manera favorable hacia los futuros profesionales o simplemente el cierre definitivo de la Institución.

Por ello, se regularon en todos los medios que hubo falencias y se analizaron los riesgos dentro de la infraestructura académica, la cual se haría estudios indispensables que fundamente si 
esta apta para el desarrollo educativo de acuerdo con los estatutos de control de la calidad académica para gestionar con mejor eficacia a los estudiantes cuyo estándares intenta cambiar con el impacto que concluyó (CES, 2014) al cuestionar la calidad de la Universidad de Guayaquil:

"La calidad de la Universidad de Guayaquil ha sido severamente cuestionada por los informes preliminares del CEAACES, organismo que ha calificado mayoritariamente de insuficientes a los indicadores de los 4 criterios en los que se basa el modelo de evaluación y acreditación universitaria. Es una realidad que las dimensiones relacionadas con la académica, la eficiencia académica, la investigación, la organización e infraestructura institucional deben ser transformadas de forma estructural a partir de la configuración de un nuevo proyecto institucional basados en un modelo de gestión sistémica y por procesos de mejora continua." (CES, 2014)

Luego la Universidad es intervenida y se procede a reformar el Currículo y se adopta el Modelo Educativo Ecológico, el diseño de un modelo académico para la UG debe integrar las definiciones del Sistema de Educación Superior se rige por principios de autonomía responsable, cogobierno, igualdad de oportunidades, calidad, pertinencia, integralidad y autodeterminación para la producción del pensamiento y conocimiento en el marco del diálogo de saberes, pensamiento universal y producción científica tecnológica global. Estos principios rigen de manera integral a las instituciones, actores, procesos, normas, recursos, y demás componentes del sistema, en los términos que establece esta Ley (Art. 12 LOES, Principios del Sistema). En este sentido, la Universidad de Guayaquil, deberá: a) Asumir los principios del Sistema de Educación Superior establecidos por la Constitución de la República del Ecuador y la Ley Orgánica de Educación Superior y armonizar con ellos las proyecciones académicas de la UG. 
b) Promover la formación integral del talento humano orientado a la investigación para fomentar el desarrollo de los procesos de investigación acorde a los avances técnicos, científicos y tecnológicos.

c) Responder a las expectativas y necesidades de la sociedad en la prospectiva del desarrollo científico, humanístico y tecnológico y a la diversidad cultural.

d) Articular la oferta docente en relación a la pertinencia y necesidades de desarrollo local, regional; promover la diversificación de las profesiones de acuerdo a las tendencias de mercado, demográficas espacio ocupacional, promoviendo la matriz productiva y cognitiva.

e) Armonizar las funciones sustantivas en orientación a desarrollar una estructura productiva.

f) Promover un proceso formativo que vincule al estudiante en mantener el conocimiento efectivo y eficiente en los diferentes ámbitos de la ciencia.

g) Promover gestionar el conocimiento en armonización con los procesos de investigación y de vinculación.

h) Garantizar un perfil de egreso que responda a las necesidades del entorno y un perfil profesional orientado a la prevención y solución de la problemática social.

Componentes del Modelo Educativo de la Universidad de Guayaquil

- La sociedad que se espera construir y transformar

- Los sujetos ciudadanos e interculturales que se van a formar 
Evelyn J. Henríquez-Antepara; Ruth E. Paredes-Santín; Oscar O. Apolinario-Arzube

- Los conocimientos que se organizan para la resolución de problemas: convergencia metodológica

- Los aprendizajes basados en la praxis de investigación

- Estrategias, ambientes, contextos de aprendizajes

- Gestión social del conocimiento

- Funciones y perfil del profesor investigador

- Principios y valores humanos y ciudadanos

El principal objetivo de la Carrera para los futuros profesionales durante el proceso educativo en la Universidad de Guayaquil, es procurar que se desenvuelva en todos los ámbitos, acoplándose día a día en el desarrollo de nuevas ciencias y tecnologías que influya a las masas para tener competencias profesionales en el mercado laboral, con un perfil de liderazgo, creatividad, poder de análisis capacidad de adaptación a nuevos ambientes, toma de decisiones, seguros de quienes son y hacia donde desean llegar porque poseen los competencias que necesitan, lamentablemente en la actualidad se vive una crisis de conocimiento en dicha Institución.

\section{Análisis de las Competencias Profesionales en los Universitarios para la Inserción Laboral.}

\section{Competencias Profesionales}

Las competencias profesionales son el conjunto de habilidades que poseen las personas y que deben aplicarlas de la forma óptima en cada lugar que de desempeñe su ejercicio laboral, un sector o una actividad determinada en un tiempo específico, no solo son competencias netamente cognitivas, 
sino todas aquellas que forman parte de la personalidad del ser humano, esto implica que debemos identificar al profesional de forma integral, holístico con competencias específicas y genéricas que deben ser desarrolladas en su totalidad en la universidad.

Aptitud de una persona para desempeñarse en el área laboral con base a los conocimientos y capacidades en el saber, el hacer y el saber hacer adquiridos a lo largo de los estudios académicos.

“Aptitud de un individuo para desempeñar una misma función productiva en diferentes contextos y con base en los requerimientos de calidad esperados por el sector productivo. Esta aptitud se logra con la adquisición y desarrollo de conocimientos, habilidades y capacidades que son expresados en el saber, el hacer y el saber hacer.” Instituto Tecnológico de Sonora

Es indisociable de la noción de desarrollo y de aprendizaje continuo unido a la experiencia. Constituye un capital o potencial de actuación vinculado a la capacidad de movilizarse o ponerse en acción.

Se inscribe en un contexto determinado que posee unos referentes de eficacia y que cuestiona su transferibilidad en su puesto de trabajo. Lo que dio lugar al enfoque de competencias centrado en la tarea. En un siguiente momento se prestó atención a los rasgos y características del profesional excelente, aquel capaz de dar el máximo resultado en su actividad profesional, aquellas competencias que diferenciaban entre el profesional que cumplía con su tarea y el profesional que destacaba en dicho logro. En este nuevo enfoque centrado en el perfil no sólo se prestaba atención a la base técnica y profesional sino que se empezó a indagar acerca de las competencias clave, aquellas competencias dotaban de flexibilidad, capacidad de aprendizaje y superación, y todos aquellos rasgos que se estimaran necesarios para llegar a ser este tipo de profesional excelente. 
La necesidad de considerar la complejidad de las competencias y su clasificación en específicas y genéricas o transversales es destacada por diferentes autores y contemplada en los procesos de reforma curricular que se desarrollan en la universidad actual (Tuning, 2003). Le Boterf (2001), al referirse a los diferentes tipos de competencias plantea, además de las competencias técnicas referidas al «saber y el saber hacer» en una profesión específica, competencias sociales, relacionadas con el «saber ser» profesional. En esta misma línea, Bunk (1994) refiere, además de las competencias técnicas que acabamos de mencionar, otros tipos de competencias profesionales de carácter general tales como:

Las competencias metodológicas, relacionadas con la capacidad de transferir el «saber hacer» a diferentes contextos profesionales.

Las competencias sociales, referidas a las habilidades comunicativas y de interacción social.

Las competencias participativas, referidas a la pertenencia a un grupo, a la toma de decisiones y la asunción de responsabilidades.

La Universidad de Guayaquil con el paso de los años ha modificado su infraestructura, pero como dato investigativo se debe cuestionar la evolución determinativa, que ha surgido en la carrera de Ingeniería en Sistemas Computacionales, al modelo de educación y además también el modelo del sistema integrado mediante (Tecnológico Nacional de México, 2008) concluye que:

“(...) tiene un enfoque basado en procesos, y determina las funciones $\mathrm{y}$ actividades relacionadas entre sí, permitiendo que los recursos y elementos de entrada se gestionen y se transformen, con el fin de satisfacer a los clientes a través del cumplimiento de sus requisitos. Este modelo conceptual se integra en un solo sistema de gestión considerando la 
Responsabilidad Social, Calidad, Medio Ambiente, Modelo de Equidad de Género, Seguridad y Salud Ocupacional.” - (Tecnológico Nacional de México, 2008).

\section{Las Competencias Profesionales, Una Mirada Internacional}

En la Universidad Tecnológica de Nezahualcóyotl (UTN) en México se realizó un estudio en donde se evaluó los conocimientos profesionales que imparte esta institución y determinar si pueden desarrollar las competencias necesarias para que un grupo de pasantes de la carrera de informática puedan desempeñarse de manera adecuada durante su estadía empresarial.

De acuerdo con La Organización para la Cooperación y el Desarrollo Económicos (OCDE) ha hecho importantes aportes en la materia y define las competencias como "la capacidad de poner en práctica de manera integrada habilidades, conocimientos y actitudes para enfrentar y resolver problemas y situaciones" (INEE, 2005:16).

Por medio de una encuesta realizada a académicos, empleadores y graduados se estableció que en el proyecto intervienen dos tipos de competencias: específicas y profesionales: la primera es relacionada con el conocimiento adquirido que aborda temas de acuerdo al perfil profesional de los estudiantes de la Carrera de Sistemas Computacionales, mientras que la otra se refiere a las capacidades que se deben desarrollar para aprender, analizar y que contribuye con en el ambiente laboral, según Tuning, la formación universitaria debe lograr un complemento entre competencias genéricas y específicas.

Durante las pasantías de los estudiantes se hizo una comparación de las competencias exigidas en el desempeño laboral y las capacidades previstas que debe tener un egresado en informática. Con respecto a las competencias específicas los pasantes demostraron un dominio 
elemental de los conocimientos básicos del área y la capacidad de manejo de información. En las competencias genéricas el trabajo en equipo, la capacidad para aprender y asimilar rápidamente los nuevos contenido y la polivalencia que indican que los estudiantes están dispuestos a desempeñarse en diferentes actividades que se requiera durante el proceso informático, todas estas obtuvieron alto puntaje pero, una de las competencias peor calificada fue la toma de decisiones, los estudiantes mostraron dificultad en analizar y dar solución a problemas presentados.

En este estudio se pudo observar que hay una deficiencia con respecto a las competencias intelectuales las cuales en el desempeño profesional son necesarias y con base a las exigencias empresariales de hoy en día señala que es importante que el estudiante adquiera competencias en donde pueda aportar soluciones a posibles problemas que se puedan presentar. Se busca mejorar la manera en que se imparte los conocimientos a los estudiantes, no solo insertar teoría, si no impulsar al estudiante a que pueda desarrollar competencias, hacerlo más activo para la sociedad, que en el momento que incursione en el mercado laboral tenga atributos que pueda aportar directamente a las exigencias que se solicitan.

Otro estudio realizado pero, esta vez en la Universidad de León en España investiga la manera de fomentar la empleabilidad de sus graduados. Debido a diferentes factores que pueden ser un obstáculo para la inserción laboral de sus estudiantes como la crisis económica, plantean dos estrategias: los planes de estudio y el apoyo en la transición al mundo laboral.

Se realizó investigaciones en las diferentes páginas web de universidades españolas y encuestas hacia aquellas entidades que se encargan de la orientación profesional de los estudiantes para analizar el término de empleabilidad y que se está haciendo como entidad educativa para 
desarrollar competencias en donde los estudiantes puedan demostrarlas en sus prácticas empresariales.

La interrogante que se plantea es: ¿De qué manera las universidades españolas han colaborado para cumplir esta tarea? En respuesta a esta pregunta, consideraremos dos puntos:

La empleabilidad de los graduados se debe trabajar en conjunto, la participación del Gobierno, futuras empresas en donde los estudiantes pondrán en ejecución los conocimientos adquiridos y por supuesto la universidad de donde salen estos profesionales, esta última no deja de desempeñar un rol importante en el camino de los estudiantes, ya que la universidad contribuye en el desarrollo de las competencias que puedan dar respuesta a las necesidades que pide el mercado laboral.

Los planes de estudios son la fuente de conocimiento para los universitarios, por lo tanto, para el desarrollo de estos se debe tomar en cuenta lo que realmente exigen y requieren los empleadores, elaborar contenido que ayude al estudiante a desarrollar competencias y capacidades que aporten en el desempeño profesional y se ajusten a las demandas del mercado laboral.

El mercado laboral cada vez exige más de sus trabajadores, hoy en día un título universitario no garantiza un puesto de trabajo, por consiguiente la preparación constante por parte del estudiante es la clave para que pueda sobresalir.

La universidad juega un rol importante en la preparación de sus estudiantes, a veces no actualizar la malla curricular para que vayan acorde con las necesidades que exige la sociedad, provocan un desequilibrio en el momento que el universitario incursiona en la búsqueda de empleo, 
por lo tanto se considera que los conocimientos impartidos en los centros de estudio muchas veces no abarcan con todas las expectativas empresariales.

La falta de gestiones académicas para conseguir un compromiso Gubernamental-Empresarial ha dinamizado el desempleo de un recién graduado, y si a esto le sumamos el temor del estudiantes al no sentirse totalmente preparados para las Prácticas Profesionales daría un resultado poco alentador para las futuras generaciones.

Al analizar las competencias profesionales que existe entre los estudiantes, se demuestra una gran falencia o determinación de proseguir con su carrera de estudio; y de acuerdo con los diferentes análisis e hipótesis se concluyó que en la actualidad las capacidades y habilidades cognitivas con las que cuenta un estudiante ha decaído gradualmente al postularse en el medio laboral, a veces no cumplen con lo requerido, por ello su rendimiento decae considerablemente, esto dispondría a resolver los diferentes requerimientos dado por una empresa, en conclusión esto se debe, a la falencia en su formación académica que presentan poco interés en aprender un poco más sobre algún tema alterno a su educación, o en ocasiones el escaso el tiempo disponible de estudio, dando la posibilidad de aprender por otros medios.

El futuro de los profesionales deberá de demostrar al mercado competitivo, las diversas competencias que ha obtenido a lo largo de su jornada estudiantil, en la cual juegan un papel muy importante en días actuales, debido a esto, será para postularse a un puesto de trabajo, el mismo deberá de demostrar sus atributos y conocimientos adquirido, por decir: iniciativa al realizar cualquier tipo de actividad, empatía hacia sus compañeros de trabajo, flexibilidad en el tiempo o de cualquier ámbito que genere la empresa, trabajo en equipo, ser eficiente al poder realizar cualquier 
inconveniente o problema que se le ha encomendado y ser capaz de adaptarse al entorno de trabajo, lo cual le permitirá desenvolverse mejor en las actividades encomendadas.

Sin embargo, al hacer una comparación entre las capacidades que adquiere con las competencias que encuentra en el ámbito laboral puede originar que el profesional sienta que no está preparado para desempeñar dichas responsabilidades. Por otro lado, es necesario que exista ese compromiso de ambas partes tanto del estudiante como el docente dentro de la Institución de Educación Superior, esto permitirá que el estudiante muestre interés por aprender más acerca de lo que se imparte en clases. Además, que esto beneficiara al futuro profesional de manera que estas aptitudes y habilidades crezcan generando nuevos conocimientos, que le permitan estar al mismo nivel de las competencias actuales.

Para esto, debemos de conocer si la Carrera de Ingeniería en Sistemas Computacionales implementa o gestiona el desarrollo de la Tecnologías de la Información y la Comunicación (TIC), además hay q ver si implementa las condiciones de estudio necesaria, así como lo implementa otras Instituciones Educativas Nacionales e Internacionales.

La reforma curricular universitaria que tiene lugar en la actualidad en el proceso de convergencia europeo de Educación Superior, dirigido a lograr competitividad, empleabilidad y movilidad para los profesionales en Europa a partir de titulaciones fácilmente comparables y comprensibles, centra la atención en la formación en competencias profesionales reconoce su carácter complejo y las clasifica en dos tipos fundamentales: (Tempus, 2003)

Genéricas Genéricas (transversales, comunes a todas las profesiones). En estas competencias se incluyen elementos de orden cognitivo y de orden motivacional, y se expresan a través de las denominadas: 
Evelyn J. Henríquez-Antepara; Ruth E. Paredes-Santín; Oscar O. Apolinario-Arzube

- Competencias instrumentales, de orden metodológico o de procedimiento, tales como la capacidad de análisis y síntesis, de organización y planificación, y de gestión de información.

- Competencias personales, tales como la capacidad para el trabajo en equipo, la habilidad para el manejo de las relaciones interpersonales, el compromiso ético.

- Competencias sistémicas, que se manifiestan en el aprendizaje autónomo, la adaptación a nuevas situaciones, la creatividad y el liderazgo, entre otras.

\section{Específicas (relativas a una profesión determinada).}

El Proyecto Tuning América Latina se inicia en el año 2004 con el objetivo de propiciar la reflexión y el intercambio entre los profesionales de la Educación Superior en América Latina, desde una posición de respeto a la autonomía y diversidad de cada región y cultura académica, en torno a la mejora de los procesos de enseñanza y aprendizaje universitarios orientados a la formación integral del estudiante desde un enfoque de competencias. Entre sus primeras tareas se plantea la identificación de las competencias genéricas para América Latina, y para ello se toma como referencia el listado de las treinta competencias genéricas identificadas para Europa, como resultado de lo cual se llega a la identificación de veintisiete de tales competencias. La importancia de las competencias genéricas en la formación actual del profesional es destacada en el Informe final Proyecto (Tuning, 2008), cuando se plantea:

Los campos profesionales se transforman y se generan nuevos nichos de tareas $y$, paralelamente, anulan o disminuyen las posibilidades de otros trabajos. La mayor parte de los estudios recientes señalan que una persona cambiará varias veces de empleo durante su etapa laboral activa. Por lo tanto, la versatilidad es, cada vez más, una característica fundamental 
para desarrollar en la formación profesional. Es decir que la flexibilidad mental, la capacidad para adaptarse a nuevos desafíos, el saber cómo resolver problemas y situaciones problemáticas, la preparación para la incertidumbre son las nuevas habilidades mentales que requerirán los profesionales del mañana y en las que debemos entrenarlos. Se hace necesario patrocinar una formación que permita realizar ajustes permanentes, demostrar equilibrio ante los cambios y capacidad de inserción ciudadana en contextos de vida democráticos. (Viviana González \& González Tirados, 2008)

En Ecuador tenemos la Universidad de Guayaquil como la entidad educativa de mayor relevancia en el país por el volumen de sus estudiantes.

“La institución es una de las más grandes del país, con cerca de 60000 estudiantes. En el 2013 bajó de la categoría B a la D. Poco antes fue intervenida por el CES debido a una serie de denuncias de irregularidades académicas y administrativas. Según el Reglamento para la Determinación de Resultados del Proceso de Evaluación, Acreditación y Categorización de las Universidades y Escuelas Politécnicas, regulado por el CEAACES, las instituciones ubicadas en la última categoría debían cumplir con un plan de fortalecimiento institucional que les permita ubicarse, al menos, en la categoría C. La U. de Guayaquil superó ese pronóstico y retomó su ubicación en la B.” - (El Comercio, 2016)

\section{Posición científica personal referente al tema}

Compartimos con (..) “Universidad de Granada (MARTÍNEZ, 2002), destaca que los empresarios echan en falta una serie de conocimientos, no vinculados directamente al ámbito académico, sino más relacionados con lo que se está denominando competencias profesionales, 
como son la capacidad de integración, de adaptación al cambio, polivalencia, movilidad, disponibilidad, implicación y Compromiso con su puesto de trabajo y con la empresa."

Es importante reflexionar que el perfil del profesional que exige la sociedad globalizada debe ser integral: sus conocimientos que son los saberes impartidos en las instituciones educativas, “el saber hacer" la forma como se transferir los conocimientos en la práctica y desempeño de las actividades asignadas, la forma de contribuir para un clima laboral estable y armónico, el saber tomar decisiones acertadas, la adaptación al cambio como parte del crecimiento profesional, el sentido de pertenencia en la organización y la actitud q son las características que buscan las empresas en sus colaboradores, es la obligación y desafío que tienen las instituciones de Educación superior.

La Educación Superior es transcendental en la sociedad de un país, las universidades son consideradas la sociedad del conocimiento, pero que sucede con las materias que se imparten en 1 Carrerea de Sistemas Computacionales, será que van de la mano con la las exigencias actuales de las economías globales, para saberlo debemos revisar la malla curricular de la carrera la cual está compuesta por:

\begin{tabular}{|c|c|c|c|}
\hline $\begin{array}{c}\text { EJES DE } \\
\text { FORAMACIÓN }\end{array}$ & ASIGNATURAS & CRÉDITOS & \% \\
\hline Básicas & 20 & 87 & 37,339 \\
\hline Humanísticas & 7 & 21 & 9,013 \\
\hline Profesionales & 25 & 110 & 47,210 \\
\hline Optativas & 4 & 12 & 5,150 \\
\hline $\begin{array}{c}\text { Servicio } \\
\text { Comunitario }\end{array}$ & 1 & 3 & 1,288 \\
\hline Total & $\mathbf{5 7}$ & $\mathbf{2 3 3}$ & $\mathbf{1 0 0 \%}$ \\
\hline
\end{tabular}

Tabla No 1.- “Tabla de ejes de formación de la carrera de Ingeniería en Sistemas Computacionales"

Fuente: Sistema integrado de la Universidad de Guayaquil 
La Carrera de Ingeniería en Sistemas Computacionales tiene 1972 estudiantes cuenta con 3 laboratorios con 40 computadoras, estos laboratorios no son suficientes se necesitaría 8 laboratorios para cubrir con este número de estudiantes, los docentes tratan de suplir esta debilidad aplicando metodologías que reemplacen las clases prácticas por deberes de investigación y horas autónomas, asignándoles al estudiante la responsabilidad de nutrirse de los conocimientos que nos pueden ser transferidos en el aula de clases, deben ellos adquirirlos de forma independiente, esta debilidad influye en el desarrollo de las competencias profesionales específicas creando una desventaja frente al profesional de las instituciones privadas, pero es la realidad con la que cuenta la Universidad de Guayaquil.

Desde esta perspectiva el currículo universitario está orientado hacia cuatro funciones sustantivas:

- Formar personas y ciudadanos capaces de preservar y co-crear la cultura

- Indagar, explorar y adquirir conocimiento, producir e interpretar significados a través de esquemas de extensión y profundidad.

- Re-crear, integrar e innovar el conocimiento construyendo objetos complejos, creativos y cambiantes.

- Transformar la realidad a través de la experiencia de aprendizaje y gestión de saberes de los sujetos educativos.

Encarnación Soto plantea cuatro principios en su ensayo sobre las "escuelas aceleradas", que se despliegan como sustento de su planteamiento filosófico educativo, cuya intencionalidad es construir una nueva mirada pedagógica centrada en la autoestima, el 
Evelyn J. Henríquez-Antepara; Ruth E. Paredes-Santín; Oscar O. Apolinario-Arzube

Aprendizaje relevante, proyectos conjuntos y autonomía con responsabilidad, con miras a "Crear espacios educativos donde cada uno ha de tener la oportunidad de sobresalir como miembro creativo, crítico y productivo de la sociedad" (Soto, 2010)

Si concertamos nuestro pensamiento a lo planteado por (Noro, 2016), al realizar un análisis sobre la escuela del presente y del futuro y el significado de la Globalización, enmarcado en las ideas o fundamentos de la realidad podremos apreciar como existen temas señalados por este autor muy coincidentes con nuestros puntos de vistas y sustantivamente importantes. Enfatiza Noro, en que...." la educación permite observar y ayudar a construir una nueva realidad, de un mundo más armónico y habitable, incluida la presencia de los seres humanos. Señala que podemos caminar hacia un futuro mejor..."

Es entonces perfectamente entendible que la globalización marca una "Nueva Era", donde surgen nuevos valores, sueños, formas de organizar los conocimientos, de surgimientos de nuevas relaciones sociales, nuevas formas de dialogar con la realidad, nuevo modo de experimentar la última realidad y nuevas maneras de entender al ser humano en el conjunto de seres..." (Noro, 2016)

\section{De la crisis a la oportunidad, posibles soluciones}

Después de analizar la problemática existente consideramos que las posibles soluciones estén en:

- Revisar y actualizar la malla con asignaturas que contribuyan con el perfil integral y competente del profesional bajo las nuevas exigencias de la sociedad globalizada

- Las asignaturas que están orientada al desarrollo de las competencias profesionales genéricas deben interactuar con el resto de las asignaturas. 
- Los docentes deben trabajar en conjunto reflexionando que el profesional debe ser integral, por lo que deben motivar al desarrollo de las competencias profesionales desde cada una de las asignaturas.

- Se debe crear grupos de investigación científica desde los primeros semestres, para cultivar la investigación y creación de nuevos proyectos, que deben ser plasmados en artículos científicos.

- La tesis o proyectos deben ser un compendio de artículos científicos y por lo menos dos SCOPUS, así se evidencia una verdadera investigación que ayuda al estudiante en el desarrollo e transferencia de los saberes recibidos sino, contribuye a la Carrera para ascender a la categoría A

- Interdisciplianriedad y transdissciplinariedad como parte de nuevos paradigmas integradores que no se evidenció en los procesos andragógicos.

Se considera muy urgente la implantación de talleres, clubs, o consejos estudiantiles, que aporten con sus conocimientos a manera de apoyo, pues al contar con una gama de especialidades, conocimientos actuales-constantes, investigaciones de campo y equipos adecuados para este efecto, el recién graduado no saldría tan solo con la idea de buscar un trabajo, sino que estaría preparado para lanzar sus nuevos proyectos y enfrentarse al mundo empresarial con una verdadera actitud de liderazgo.

Todas estas interrelaciones estudiantiles, nos ayudarían a identificar mucho más a fondo las aptitudes del estudiante para así poder explotar más a fondo sus capacidades, pues está comprobado que una excelente nota no define a un buen profesional, porque al final lo que cuenta son los 
Evelyn J. Henríquez-Antepara; Ruth E. Paredes-Santín; Oscar O. Apolinario-Arzube

conocimientos, las practicas que hayas tenido y el desenvolvimiento, que probablemente no muchos tiene la oportunidad o facilidad de adquirirlo o en su defecto trasmitirlo.

\section{Conclusiones.}

Resulta evidente la importancia y pertinencia del tema tratado, ya que un abordaje científico condiciona una mejor praxis en el desarrollo de las competencias profesionales en los alumnos que transitan por la Educación Superior, logrando un egresado más competente y preparado para enfrentar el mundo globalizado cumpliendo con las exigencias de la nueva sociedad.

Se pudo constatar que existe una crisis en el desarrollo de las competencias profesionales en los alumnos que de la carrera de Ingeniería en Sistema Computacionales, evidenciando la necesidad de buscar alternativas de solución que se enmarquen entre otros aspectos en el trabajo Interdisciplinario y transdisciplinario.

La malla contiene asignaturas que mantienen unidades desactualizadas en respuestas a las exigencias de la sociedad globalizada, las asignaturas no se encuentran aisladas en su proceso académico, no existe trabajo colaborativo como metodología integradora.

\section{Bibliografía.}

CES. (2014). Plan Excelencia Universitaria. Quito: Consejo de Educación Superior Ecuador.

CONEA. (2009). Mandato Constituyente No. 14: La evaluación de desempeño institucional de las IES. Quito: Consejo Nacional de Evaluacion y Acreditacion dela Educación Superior.

Diaz Barriga, Á. (2003). La Investigació Curricular en México, en la Década de los noventa. Perfiles Educativos, 129-133.

El Comercio. (6 de Septiembre de 2016). La Universidad de Guayaquil pasa de la categoría D a la B. Obtenido de elcomercio.com: http://www.elcomercio.com/actualidad/universidad-guayaquilcategoria-educacionsuperior-reneramirez.html 
Larrea, E., \& Montalvan, M. (2016). Modelo Educativo Ecológico de la Universidad de Guayaquil. Modelo de Formación Integral Orientado a la Investigación y Gestion Social del Conocimiento, 6-15.

Moreno Moreno, M., \& Muñoz García, A. (2007). Bases psicológicas para una propuesta de ambientación del curriculum. Revista Iberoamericana, 10(1), 239-246.

Soto, E. (2010). Escuelas Aceleradas. Documento Policopiado. Máster + Doctorado en Políticas y Prácticas de Innovación Educativa para la Sociedad del Conocimiento Universidad de Almería-UCSG. Universidad de Guayaquil: Guayaquil.

Tecnológico Nacional de México. (2008). Sistema de Gestión Integral. Tabasco: Instituto Tecnológico Superior de Centla.

Tempus. (2003). Una introducción a Tuning Educational Structures in Europe. La construción de las universidades al porceso de Bolonia. Socrates.

Tuning. (2008). Proyecto Tuning América Latina. Deusto: Alfa.

Viviana González, M., \& González Tirados, R. (2008). Competencias genéricas y formación profesional: un análisis desde la docencia universitaria. Revista Iberoamericana de Educación, 47(1), 185-209. 